\title{
An Effective Approach to Avert Wormhole Attack In AODV
}

\author{
Kollu Spurthi ${ }^{1}$, T.N. Shankar ${ }^{2}$ \\ ${ }^{1}$ Research Scholar, Dept of CSE, Koneru Lakshmaiah Education Foundation, Vaddesvaram,Guntur,AP,India, \\ Kolluspoorthy03@gmail.com \\ ${ }^{2}$ Professor, Dept of CSE, Koneru Lakshmaiah Education Foundation, Vaddesvaram, Guntur, AP, India, \\ tnshankar2004@rediffmail.com
}

\begin{abstract}
One of the obvious zone attracting researchers with its self-configuring dynamic nature in the various areas like military applications, recovery of disaster, gaming, etc. is none other than MANETS. It extends their scope for researches as the characteristics of MANETS by itself entail security threats. These Adhoc networks being subjected to a larger domain of security threats give an enumerated path for research. Among the security threats prevailing. Wormhole attack is one of the interesting zone possible to opt. In this paper, we take the privileged of dealing with wormhole attack characteristics, their impact on the network, and thereafter put forth a well-defined algorithm and implement the security-based framework which entails all the detection features for handling wormhole attack in AODV.
\end{abstract}

Key words: MANET; Network security; Wormhole attack; Secured algorithm.

\section{INTRODUCTION}

Manets allude to a group of nodes that communicate with each other to share information..Manets are wireless structure less decentralized mobile network supporting numerous demands of defense handling adverse situations of natural calamities supporting individual networks is subjected to vulnerabilities from all its attackers. The dynamic and self-configuring characteristics of Manets fabricate them to security threats. Despite innumerable threats, Manet is always covered with its first shied layer of the intrusion detection system. An IDS serving its master to the fullest is still at risk of harmful attacks. The reason entailing the above situation is that the Manet needs to deliver its two responsibilities of a router and a host. The core concept of manet includes routing protocols which play a vital role in the exchange of information between nodes.

Depending on the scenario protocols can be distinguished as

- source initiated,(sender -initiated)

- table-driven(two-dimensional structure)

○ hybrid (combinational approach)
AODV: a responsive controlling tradition, DSR: proactive arranging They are accountable for seeing the perfect path from a source center point to an objective center point in a particular MANET. One can depict arranging traditions as open, proactive, and crossbreed organizing traditions. For example, ZRF: a mutt planning custom. AODV is a blend of DSR and DSDV protocols that uses the on-demand technique. It builds a path from source to the destination when required using RREQ and RREP packets. Every RREQ sustains a time to live (TTL) value that elucidates for how many nodes this data should be delivered. This value is set to a threshold value at the beginning of the transmission and incremented at retransmissions. Retransmissions occur if no answer is received. RREP - Once the transmitted packet reaches the destined receiver using address requested or a valid route, it initiates an RREP message which is unicasted to the source of an RREQ. This acknowledgment back to the sender is made possible as every route that pushes an RREQ promises to catch back to route to the initiator. Link breakage is notified by RERR. Manets are prone to a different type of attacks by their wireless infrastructure, node communication play a crucial role in sending and receiving packets. Attacks mainly come out with malicious nodes that are of two types: Passive, Active. The active attack includes routing attacks that mainly comprise wormhole, blackhole. As the entire infrastructure of manet depends upon routing algorithms for sharing information routing attacks 1 ay a pivotal role in weakening the performance of Manet. Wormhole attacks with its pair of warriors called wormhole nodes which are joined with each other using its tunnel, hence attack is conversely named as tunneling attack. In this attack as soon as the communication channel is established the attacker listens to the communication tracking it and thereby forwarding it to the partner via the tunnel.

This attack is possible as the characteristics of attacker nodes permit them to make tunnel even for unaddressed packets and establish route which is smaller than the original one, thereby false promising as the shortest path for legal nodes. Hence this attack is proving to be harmful to both reactive and proactive protocols. Depending on 
the various factors like implementation of attacker nodes, method of attacking, medium preferred, visibility, placement of identity in packet header wormhole attack is characterized as open/exposed, hidden/closed, semi-open.

The basic blend of this paper is to detect and avoid a wormhole attack using the AODV routing protocol, and every packet is encrypted with a tiny encryption algorithm This proposed technique is evaluated with the existing methodology.

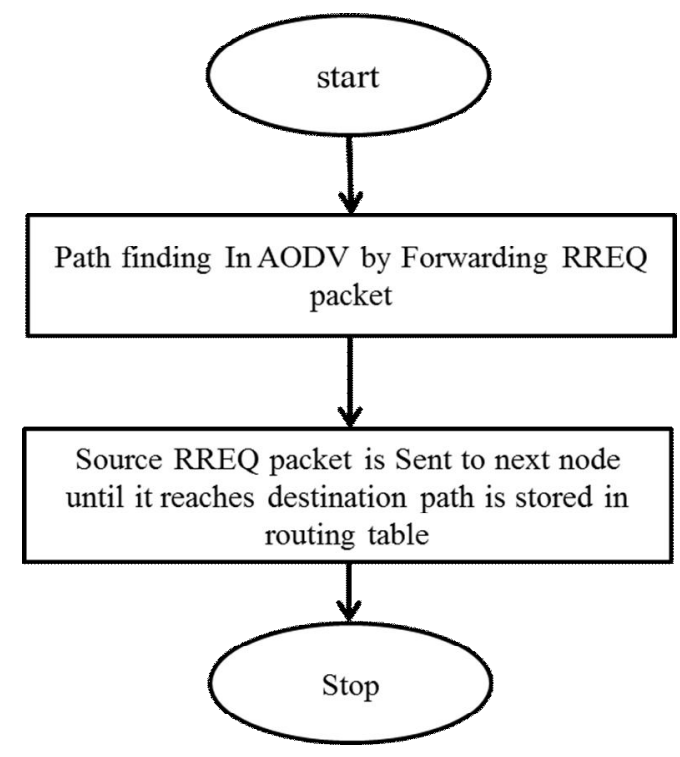

Figure1: Flow chart indicating AODV working

Working of AODV routing algorithm includes the following steps:

Deciding path:

Present AODV protocol mainly concentrates on learning roots from source node to destination node and vice-versa. It is mainly designed for supporting mobile Adhoc networks. AODV extends its routing capabilities for both unicasting and multicasting. As its name, on-demand algorithm establishes paths among nodes only when the requirement arises from the source node, and retain it until the source intends. AODV accomplish routes with the help of a query cycle including route request and route reply.

Packet transmission:

Transmission starts as soon as the path is established among source and destination nodes using the protocol AODV. The source node initiates transmitting the packets to the intended destination via the intended path by broadcasting control packets for all its neighbors. Each neighbor receiving the control packet will send it to its neighbors, at the same time it makes sure that the packet forwarding is not repeated by making use of sequence numbers. The transmitted packets finally reach its destination after which the packet is no more forwarded.

Capturing the victim / considered node:

The victim is captured by sensing that it is not forwarding the received packet to its neighbor within the stipulated time. The identification stage and suspect stage help us in confirming the victim node. These stages include the process that the source node transmits the packet to the suspected node for confirmation. Then the suspect replies appropriately to the transmitted node within time and in the proper sequence. It is considered to be a healthy node. If this does not occur, the node is assumed to be a failure node i.e., victim. This node in turn sends and gathers information from its nearby neighbor nodes. The neighboring node sends the message to the node sending the request by a suspect node replay message within the stipulated period.

As soon as a victim node is identified the source node evolves with a remedy new route for retransmitting the packets. Simultaneously, an alert message is forwarded to all nodes to make sure that no node transmits via the victim node. Sometimes, the newly discovered paths may include suspicious nodes that are identified similarly. The AODV protocol comes up with a new path. The basic criteria on which AODV protocol concentrates are non-repetition and optimal path.

AODV offers three types of control messages for maintenance of the route. A message termed RREQ i.e, the route request message is sent by a node expecting a route. AODV prefers expanding ring method during message flooding. By making use of the TTL parameter the RREQ identifies the number of hops a message needs to be forwarded. The TTL value is a predefined one initialized during the first transmission and incremented during retransmission. Retransmission is initiated when replies are not received.

Upon setting a route, data packets that are to be transmitted will be locally buffered and forwarded based on the FIFO principle. This transmission is initiated by RREQ.

\section{RELATED WORKS}

The related making shows a couple out of security strategies, for example, pre-course key checks [15], symmetric and Hitler kilter figurings, completely astounding focus based structures [8], and cream systems [14]. Before long, these systems are inadequate for dynamic structures since they require a tied down thinking (i.e., manage plan) or outside specialists (for 
instance, focal intrigue aces). In clear up a correspondence building thought for dynamic structures, directing application-level novel get-together correspondence, and unpremeditated systems connected. A tremendous proportion of procedures to empower association and play, tending to and versatility, spilled manage and the usage of affiliations are other than given. Liu et al. [18] display how managed focus focuses can self- rulingly continue and driving force with one another in a conventional (P2P) way to deal with oversee coordinate rapidly find and self technique any affiliations open on the risky condition and pass on a foreseen limit with no other individual controlling themselves in brilliant social gatherings to give higher flexibility and versatility to fiasco checking and having any kind of effect. K. Liu et al. [16] proposed TWOACK is ahead among the most essential structures for impedance ID in MANETs. TWOACK sees getting into mass interfaces by watching each datum direct over each three dynamic spotlight bases on the course from the source to the target. An unending supply of a bundle, each inside point along the course is required to send back a check bundle to the middle that is two effects from it down the course. TWOACK is required to supervise controlling customs, for example, Dynamic Source Routing (DSR). Feeney et al. [20] demonstrated Spontnet, a model utilization of a direct with no organizing system structure utility subject to the basic examinations of dynamic structures. Spontnet endowments clients (utilizing versus elucidation and short-run interface with enough clear endpoints) to fitting a gathering session key without past shared setting and to set up the shared namespace. Two applications, a key web server, and an ordinary whiteboard are given as events of system applications. They use IPSec only (utilized for Virtual Private Networks), related in spite of the web. Spotnet in like way utilizes both wired and remote affiliations and relating conventions. Ariadne [17] is an on-request arranging estimation subject to the Dynamic Source Routing (DSR) custom [2].

Problem statement:

A wormhole is one of the serious problems Manets are facing. Detection features of Wormhole are analyzed with the help of Table1.1. Applying these features, a security algorithm is introduced. The main objective of the paper is to propose a technique that identifies and detects wormhole with efficient PDR, throughput, and reduces packet drop in AODV. This technique also emphasizes vital aspects of security like confidentiality, authentication, and integrity which play a key role in securing data.

\section{EXISTING METHOD}

Blackhole attack is one more routing attack as mentioned where malicious node pulls data packets by reporting other nodes in the network that it possess the shortest path.

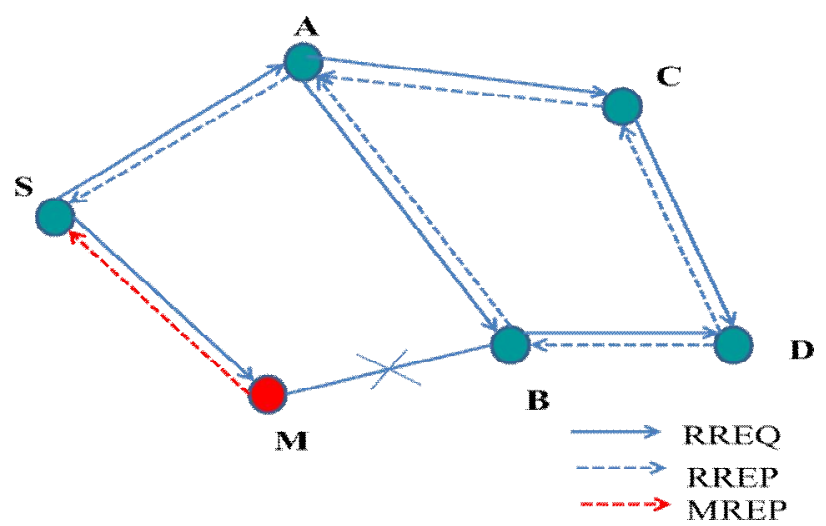

Figure 2 :Shows a black hole attack, $\mathrm{S}$ and $\mathrm{D}$ are initiator and destination nodes, $\mathrm{M}$ is the attacker node.RREP, RREQ are query cycles for finding a route from source to destination, MREP is an acronym for the malicious reply.

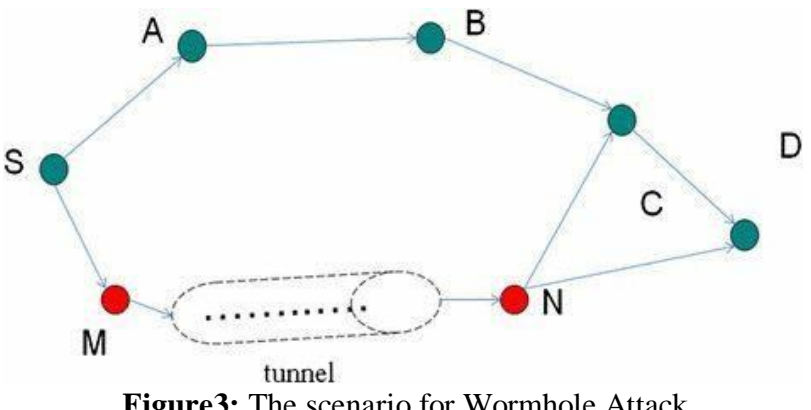

Figure3: The scenario for Wormhole Attack

"The Fig.3, above addresses the wormhole ambush situation. Here, $\mathrm{S}$ is the source center point and $\mathrm{D}$ is the objective center. $\mathrm{A}, \mathrm{B}, \mathrm{C}$ are the interfacing center points, that are giving a route between the source and the objective. $\mathrm{M}$ and $\mathrm{N}$ here are the devilish plotting center points, tunneling every one of the information and executing wormhole attack"[1].

GPS is proposed earlier to prevent wormhole attack,.GPS device is placed at every node, for the reason that location of the node is a well-chosen feature for finding wormhole,but this technique made the network high priced.

Another system was put forward to prevent wormhole using LGF protocol. This protocol operates using geo enhanced AODV .

STEP 1: The initiator node I will multicast RREQ packet to find the path to destination DE. RREQ packet contains the destination IP address, distance from the initiator node. This is sent to all the nodes that are present between the source and destination that contains the IP address of the destination. 


$$
\begin{aligned}
& \text { DIST }(I, A)=40 \mathrm{M} \\
& \text { DIST }(I, B)=53 \mathrm{M} \\
& \text { DIST }(I, E)=48 \mathrm{M} \\
& \text { DIST }(1, C)=60 \mathrm{M} \\
& \text { DIST }(B, C)=130 \mathrm{M} \\
& \text { DIST }(\mathrm{C}, D E)=180 \mathrm{M} \\
& \text { DIST }(\mathrm{D}, \mathrm{F})=45 \mathrm{M} \\
& \text { DIST }(\mathrm{I}, \mathrm{D})=62 \mathrm{M} \\
& \text { DIST }(\mathrm{E}, \mathrm{F})=85 \mathrm{M} \\
& \operatorname{DIST}(6, \mathrm{DE})=78 \mathrm{M}
\end{aligned}
$$

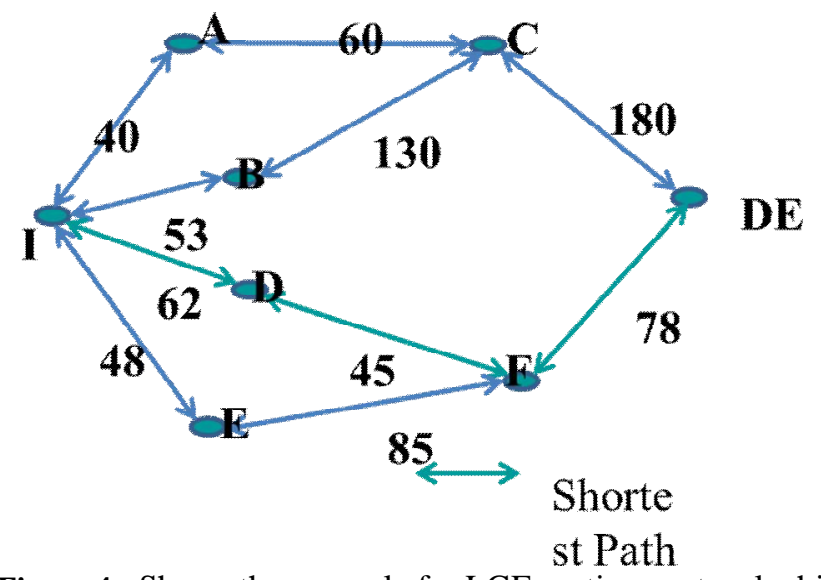

Figure 4 : Shows the example for LGF routing protocol which illustrates A, B, E, C, F AS intermediate nodes, I as the source node, DE as destination node.and the complete distance between initiator node, the destination node is $100 \mathrm{~m}$

STEP 2:RREQ packets are sent to the nodes at $100 \mathrm{~m}$ distance from the destination node since this protocol uses a free covered location tracking system. and the transitional nodes as

STEP 3:Bestowed to step 2 distance between the initiator node and destination node is evaluated.

If the distance between two nodes is within transmission range

RREQ packet is sent to a destination which in turn sends RREP packet through various intermediate nodes. Based on the distance shortest path is taken into consideration for sending data packets.

STEP 4:In case the distance is out of transmission range than the RREQ packet is discarded and an error message is sent via control packet RERR. This protocol did not prove to be efficient.

Alphanumeric routing protocol was recommended which uses the clustering technique. Here each cluster dwells master node LN, local node AN. The master node maintains routing information and is responsible for verification of every packet entering into the cluster. It forwards the packet to the local nodes. But this protocol could not prove to be strong for detecting wormhole. Here if master node security is compromised than entire cluster performance will be degraded.

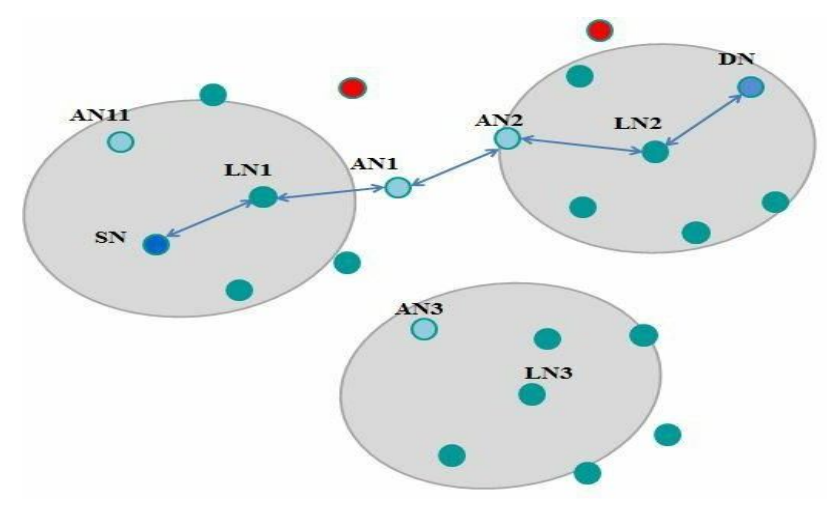

Figure 5 :shows the detailed architecture of the above routing protocol, which is divided into three clusters, red-colored nodes are considered as malicious nodes which are present outside the clusters. An Intelligent Manet algorithm was also introduced where the server agent plays a vital role. every node in the network should register with a server agent which in turn replies with a unique ID.

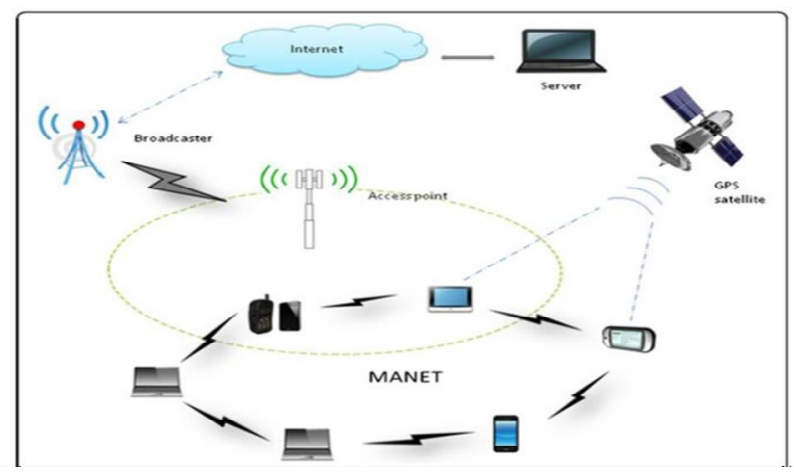

Figure 6 : Shows the intelligent manet algorithm which shows server agent, manet, and how the connection is established between them.

Latterly an approach was proposed to avert this attack. In this pitch the attack is ruled out by ensuing technique:

STEP 1: AODV establishes a path among nodes only when the requirement arises. with the help of a query cycle including route request and route reply packets.

STEP 2: Source node initiates transmitting the packets to the intended destination via the intended path by broadcasting control packets for all its neighbors. Each neighbor receiving the RREQ packet will send it to its neighbors. The transmitted packet finally reaches its destination after which the packet is no more forwarded.

STEP 3: Once the transmitted packet reaches the destined receiver using address requested or a valid route, it initiates an RREP message which is unicasted to the source of an RREQ. 
STEP 4: The traversed path is stored in the routing table

STEP 5: The traveled route in the routing table is compared against the path nodes table, if there is no similarity between tables wormhole is encountered.

STEP 6: When packets are sent from source to next hop, the packet delivery ratio is calculated.

STEP 7: RTT is calculated at every node. Accompanied by the following condition wormhole is detected

1.RTT is lesser than the threshold.

2.PDR is lesser than 1 .

3.PDR is less than 1and RTT not less than the threshold.

The above-mentioned approach is entirely pivoted on RTT and PDR, packet security is not taken into consideration. So, the proposed architecture emphasizes the important security services.

\section{PROPOSED TECHNIQUE}

The proposed approach increases the security of the packet, alongside it ensures that the integrity of the packet is maintained in the time of attack moreover detects the attack using all detection features of wormhole i.e RREQ packet, neighborhood, hop count, time. To ensure confidentiality, every packet is encrypted with TEA.

Internal programming process at node:

1. Starts configuration of nodes.

2. The node sends the request RREQ packet to next two nearby hops

3. Packet carries the TINY encryption key.

4. RREQ packet data is encrypted and sent to neighboring nodes.

5. RREP packet is received from expected nodes.Every node waits for a certain threshold time for a response from expected nodes.

NODE

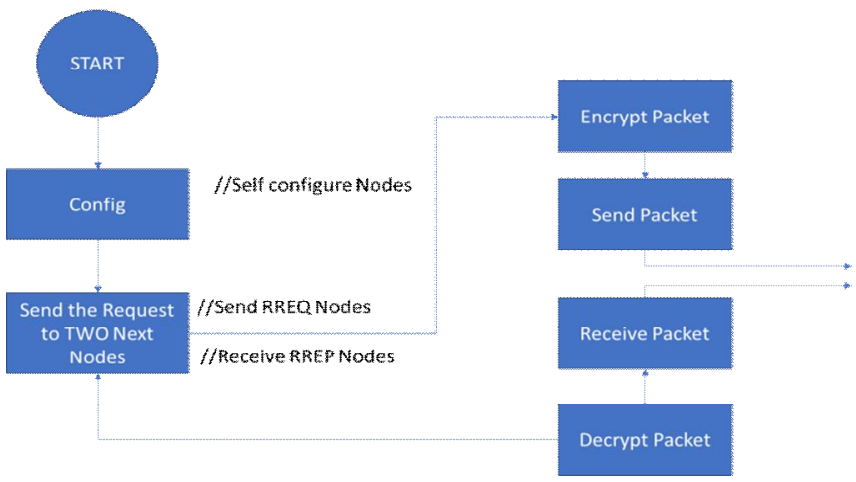

Figure7 shows the detailed process at each node.
Pseudocode for the proposed algorithm: Begin

$$
\begin{aligned}
& \text { Initialize hops } \\
& \text { Begin source and destination } \\
& \text { hops Source node }=\mathrm{IN} \\
& \text { Destination node }=\mathrm{DN} \\
& \text { For } \mathrm{i}=0 \text { to } n \text { Do For } \mathrm{j}=\mathrm{j}+1
\end{aligned}
$$

Do

$$
\begin{aligned}
& \text { IN } \square \text { sends RREQ(To establish } \\
& \text { path) (only to nearby two hops) } \\
& \text { Encrypt (packet) }
\end{aligned}
$$

Get RREP $\square$ DN(After establishing path) If(Nodes reply $<=2 *$ ts)

$$
\begin{aligned}
& \quad \begin{array}{l}
\text { Nod } \\
\text { e=Accep } \\
\text { ted Else } \\
\text { Node } \\
\text { If(routing } \\
\text { table } \\
\text { Else } \\
\text { Node= } \\
\text { If(hop co } \\
\text { nodes } \\
\text { Node= }
\end{array} \\
& \text { Else }
\end{aligned}
$$$$
\text { Node=Malicious }
$$$$
\text { If(routing table of IN==Routing }
$$$$
\text { table of DN) Node=Accepted }
$$

Node $=$ Malicious

If(hop count between

nodes matches)

Node $=$ Accepted

$\quad$ Nod
e-Malic
ious
End.

The detailed footprint of the method is explained below:

STEP 1:Every RREQ packet is encrypted with TEA(Tiny Encryption Algorithm), which can also be replaced with DES, AES.

STEP 2:RREQ packet is sent to the nearest two-hop neighbors.

STEP3: At every node traveled path is checked against path node routing table, if not similar nodes are considered as malicious.

STEP 4:Every node waits for $2 *$ ts time. where ts is the time taken for the packet to reach the next nearby hop. If it does not 
Kollu Spurthi et al., International Journal of Advanced Trends in Computer Science and Engineering, 9(5), September - October 2020,8257 - 8265

get a reply within predefined threshold time wormhole is detected.

STEP 5:Along side hop count is also taken into consideration to detect the attack. If the hop count is less than the previous path the path is checked to detect wormhole.

STEP 6:The proposed technique is verified in terms of QoS parameters using jsim.

In this process, we used Java programming language to develop a simulation process of networking for the AODV algorithm. For this simulation, a simulator class can start max 25 nodes at a time, and a random simulator selects two nodes as "malicious" nodes. All nodes start their service at a time by the simulator, The $\mathrm{X}$ node can send a packet to $\mathrm{Y}$ with the $\mathrm{Z}$ destination address. if $\mathrm{Y}$ has a relation with $\mathrm{Z}$, it sends a packet to Z. Z responds with an 'ACTCLIT' message to Y.

The $\mathrm{Z}$ routing table is given below

\begin{tabular}{|l|l|l|l|}
\hline $\begin{array}{l}\text { THIS } \\
\text { NODE }\end{array}$ & $\begin{array}{l}\text { CONNECTE } \\
\text { D NODE }\end{array}$ & $\begin{array}{l}\text { RELATION } \\
\text { OF NODE }\end{array}$ & $\begin{array}{l}\text { MY NEXT } \\
\text { NODES }\end{array}$ \\
\hline $\mathrm{Z}$ & $\mathrm{Y}$ & $\mathrm{X}$ & \\
\hline $\mathrm{Z}$ & $\mathrm{Y}$ & $\mathrm{W}$ & \\
\hline
\end{tabular}

Same way, Y also has its routing table like below

\begin{tabular}{|l|l|l|l|}
\hline $\begin{array}{l}\text { THIS } \\
\text { NOD } \\
\text { E }\end{array}$ & $\begin{array}{l}\text { CONNECTED } \\
\text { NODE }\end{array}$ & $\begin{array}{l}\text { RELATION } \\
\text { OF NODE }\end{array}$ & $\begin{array}{l}\text { MY NEXT } \\
\text { NODES }\end{array}$ \\
\hline Y & X & V & Z \\
\hline Y & W & U & \\
\hline
\end{tabular}

For this, simulation we used AODV parameters as below

AODV-NET-DIAMETER 35

AODV-NODE-TRAVERSAL-TIME 40MS

AODV-ACTIVE-ROUTE-TIMEOUT 3S

AODV-MY-ROUTE-TIMEOUT 6S

AODV-ALLOWED-HELLO-LOSS 2

AODV-HELLO-INTERVAL $1 \mathrm{~S}$

AODV-ROUTE-DELETION-CONSTANT 5

AODV-RREQ-RETRIES

AODV-PROCESS-HELLO NO

AODV-LOCAL-REPAIR NO

\section{AODV-SEARCH-BETTER-ROUTE NO}

AODV-BUFFER-MAX-PACKET 100

Table 1: Hardware and software configurations

\begin{tabular}{|c|c|c|c|c|c|}
\hline \multirow[t]{2}{*}{ Parameter } & \multicolumn{5}{|c|}{ Node Number } \\
\hline & 10 & 50 & 100 & 200 & 500 \\
\hline Area $(\mathrm{m})$ & $\begin{array}{l}300 \\
X \\
300 \\
\end{array}$ & $\begin{array}{l}900 X \\
900\end{array}$ & $\begin{array}{l}1200 \times 1 \\
200\end{array}$ & $\begin{array}{l}1500 \times 1 \\
500\end{array}$ & $\begin{array}{l}1800 X 1 \\
800\end{array}$ \\
\hline $\begin{array}{l}\text { Coverag e } \\
\text { area } \\
(\mathrm{m})\end{array}$ & \multicolumn{5}{|l|}{200} \\
\hline $\begin{array}{l}\text { Simulati } \\
\text { on Time } \\
\text { (s) }\end{array}$ & \multicolumn{5}{|l|}{8} \\
\hline $\begin{array}{l}\text { Generate d } \\
\text { message } \\
\text { number/ } \\
\text { node }\end{array}$ & \multicolumn{5}{|l|}{25} \\
\hline $\begin{array}{l}\text { Speed } \\
(\mathrm{m} / \mathrm{s})\end{array}$ & \multicolumn{5}{|l|}{$0-10$} \\
\hline $\begin{array}{l}\text { Directio } \\
\mathrm{n}\end{array}$ & \multicolumn{5}{|c|}{ Random } \\
\hline $\begin{array}{l}\text { Topolog } \\
\mathrm{y}\end{array}$ & \multicolumn{5}{|c|}{ WAXMAN } \\
\hline $\begin{array}{l}\text { Comput } \\
\text { e r }\end{array}$ & \multicolumn{5}{|c|}{ Intel i3, 16GB Ram } \\
\hline $\begin{array}{l}\text { Platfor } \\
\mathrm{m}\end{array}$ & \multicolumn{5}{|c|}{$\begin{array}{l}\text { Windows 10, 64bit Java Runtime } \\
\text { Environment }\end{array}$} \\
\hline
\end{tabular}

Implementation Results:

Figure 7.1 Shows all the 25 nodes before simulation in the network scenario. For simulation, we are using the AODV routing protocol, which starts the simulation by deciding two nodes as source and destination and starts route search process with node traverse tome of $40 \mathrm{~ms}$, and node diameter is taken as $35 \mathrm{~ms}$. Every RREQ packet has TTL which defines that the packet should reach two neighborhood nodes according to our proposed algorithm

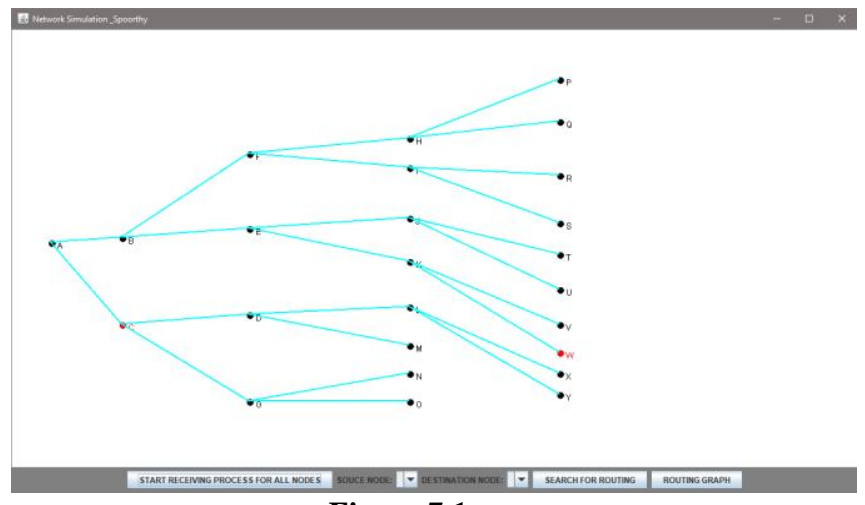

Figure 7.1 
In the below figure 7.2 two nodes are considered as source and destination, $\mathrm{A}$ and $\mathrm{K}$. The simulation process starts with the route identification process by sending the RREP packet. All nodes have their cache to maintain packet cache and routing information and buffer for a packet is given as 100 .

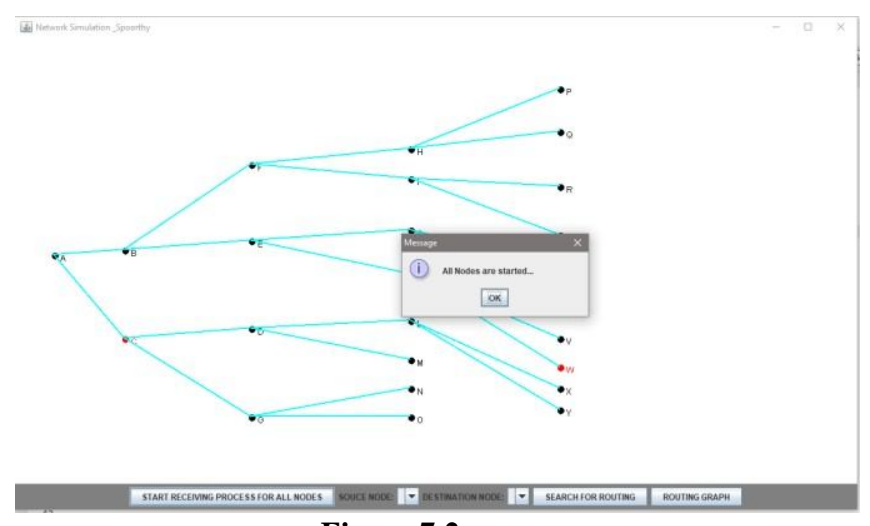

Figure 7.2

Figure 7.3 shows source node $\mathrm{A}$ and destination node $\mathrm{K}, \mathrm{W}$ as a malicious node that is selected by a simulator that causes a wormhole attack. In this phenomenon, we can choose two nodes as malicious nodes since the wormhole attack is triggered by the tunnel between two nodes.

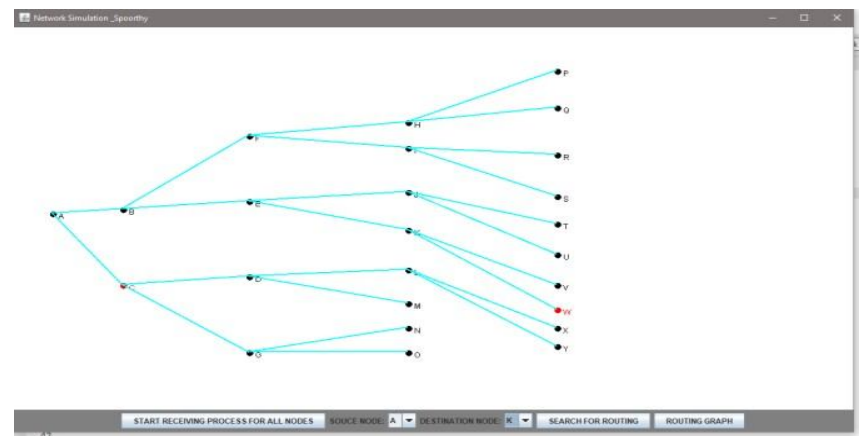

Figure 7.3

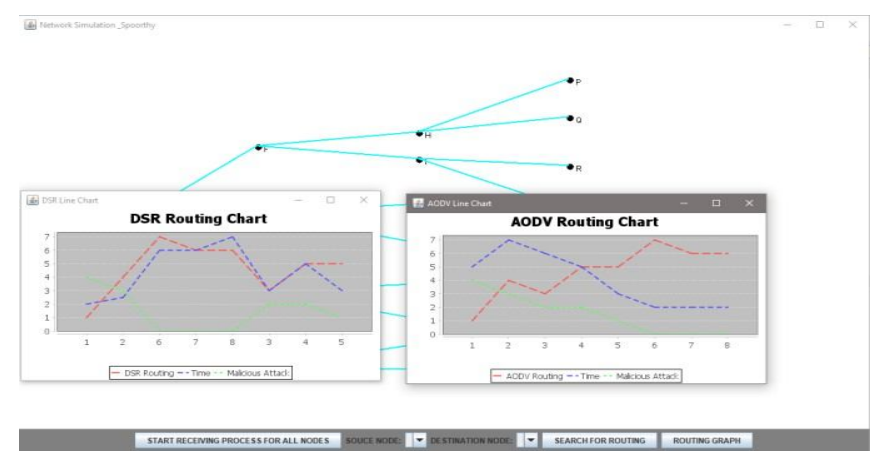

Figure 7.4

Figure 7.4 shows the Simulation Results showing fake matching which indicates the presence of malicious nodes creating a wormhole, it also shows the packet information such as source, destination, seqid, data, etc.,

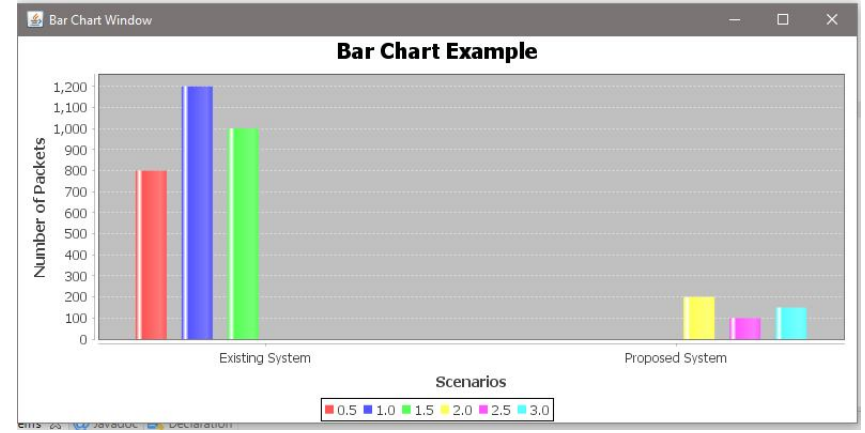

Figure 7.5

Figure 7.5 shows the Routing chart indicating routing, time, and malicious attack. The $\mathrm{X}$-axis indicates the throughput and $y$ - axis shows response count. The graph generated by simulator indicates time, route avoiding malicious nodes. In this simulation process, we compare AODV routing using a TINY algorithm with DSR. AODV routing has a positive performance than DSR when compared in terms of

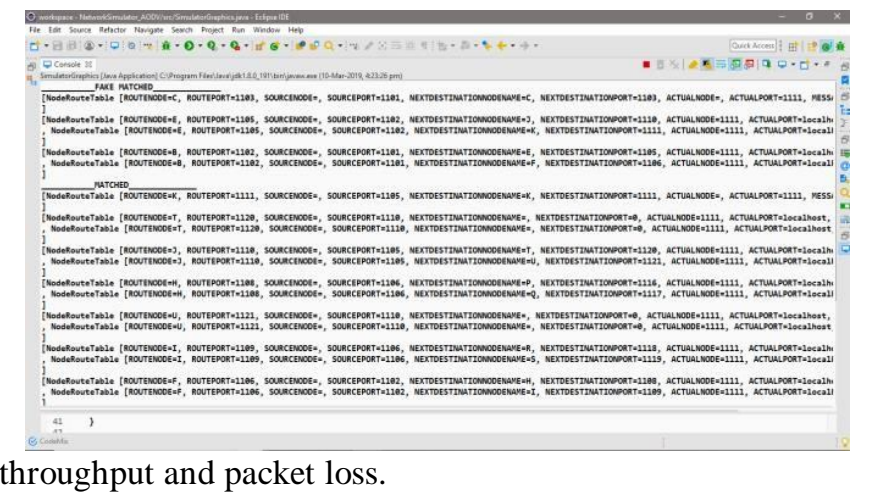

Figure 7.6

Figure 7.6 shows comparison of packet loss for an existing system with a proposed system where $\mathrm{x}$-axis shows the number of packets, the y-axis shows existing, the proposed system at different scenarios.

\section{CONCLUSION}

Mobile Ad Hoc Network (MANET) is a sort of Ad hoc encourage with versatile, remote focus focuses. Because of its exceptional characteristics like open system limit, dynamic topology trades MANET looked with an assortment of difficulties. Since every single focus point acknowledge exchanges and focus indicates are allowed to join and leave the structure, security changed into the most essential test in MANET. In this paper, a design is introduced to avoid wormhole attack and implementation is shown with the help of a java simulator. in MANET security challenges are exhibited. We also talked about routing, time, and malicious node effect on the MANET. 
Kollu Spurthi et al., International Journal of Advanced Trends in Computer Science and Engineering, 9(5), September - October 2020,8257 - 8265

\section{FUTURE SCOPE}

Starting at beginning late quickly discussed the various types of attacks MANET are facing and presented some examination regarding them. In this strategy, lessening pack overhead and overseeing time, near creating exactness is a major test. By growing precision, it can perceive fulfilling hazardous center core interests.

Finding a practical way to deal with oversee figure the limit and present astounding ID framework since reducing time and package overhead is the open edge of research in prevailing approaches. To manage this test isolating sniffing and other vanquishing approaches is recommended. MANET is a self-guided, self-configurable framework with no concentrated control. Along these lines, encryption and statement are challenging. Key scattering and control units are the most fundamental burdens. One way to deal with overseeing over through these troubles is by using bundling; in this manner, the Cluster Head can go about as the key distributor. By uprightness of MANETs dynamic topology, making and keep up packs is challenging. Using a cushy procedure for intuition [16] or swarm-based [17] is especially recommended for this test. As another examination energy, decreasing masterminding time and overseeing overhead of the encryption approach can be referenced. Overabundance structures, make gatherings of reproduced packages and waste center centers resources. In like manner, it grows block and package lost. Sensibly picking several duplicated courses, in light of hazard level, is remarkably challenging. Other than merging this system with somebody of benevolent rationality to perceive undermining center centers is another challengeable issue. Dynamic repeat is influencing in multi-type MANETs. By using this system in multi-type MANET, each inside snares its packs by sending in different frequencies. In like way, breaking one repeat does not affect others. This is a test in this strategy.

\section{REFERENCES}

1. A.Gantes and j. Stucky, "A platform on a Mobseile Ad hoc Network challenging collaborative gaming," international symposium on collaborative technologies and systems, 2008.

2. K.U. R. Khan, R. U. Zaman, and A. V. G. Reddy, "Integrating Mobile Ad Hoc Networks and the Internet challenges and a review of strategies," presented at the 3rd International Conference on Communication Systems Software and Middleware and Workshops, COMSWARE, 2008.

3. M.Suguna and P. Subathra, " Establishment of stable certificate chains for authentication in mobile ad hoc networks," presented at the International Conference on Recent Trends in Information Technology (ICRTIT), 2011.

4. H.Nishiyama, T. Ngo, N. Ansari, and N. Kato, "On Minimizing the Impact of Mobility on Topology Control in Mobile Ad Hoc Networks," Wireless Communications, IEEE Transactions, 2012.

5. F.D. Rango, M. Fotino, and S. Marano, "EE-OLSR: Energy Efficient OLSR routing protocol for Mobile Ad-hoc Networks," presented at the Military Communications Conference, MILCOM, 2008.

6. K.Du and Y. Yang, "Policy-Based Time Slot Assignment algorithm in a MANET(PBTSA)," presented at the 3rd International Conference on Anti-counterfeiting, Security, and Identification in Communication, ASID, 2009.

7. R.H.Jhaveri, S. J. Patel, and D. C. Jinwala, "DoS Attacks in Mobile Ad Hoc Networks: A Survey," presented at the Second International Conference on Advanced Computing \& Communication Technologies (ACCT), 2012

8. R.Sheikh, M. S. Chande, and D. K. Mishra, "Security issues in MANET: A review," presented at the Seventh International Conference On Wireless And Optical Communications Networks (WOCN), 2010.

9. H.Deng, W. Li, and D. P. Agrawal, "Routing security in wireless ad hoc networks," Communications Magazine, IEEE, 2002.

10. Y.Z.a and W. Lee, "Intrusion Detection in Wireless AdHoc networks," presented at the 6th Int'l. Conf. Mobile Comp. Net., MobiCom, 2000.

11. F.S.a and R. Anderson, "The Resurrecting Ducking: Security Issues for Ad-Hoc Wireless Networks," 7th Int'1. Wksp on Security Protocols. Proc., LNC, 1999.

12. X.Zhao, Z. You, Z. Zhao, D. Chen, and F. Peng, "Availability Based Trust Model of Clusters for MANET," presented at the 7th International Conference on Service Systems and Service Management (ICSSSM), 2011.

13. E.C.H.Ngai and L. M. R, "Trust and clustering-based Authentication Services in Mobile ad hoc networks," presented at the proceeding of the 24th international conference on Distributed Computing Systems Workshops 2004.

14. W.Lou, W. Liu, and Y. Fang, "SPREAD: enhancing data confidentiality in mobile ad hoc networks," presented at the Twenty-third AnnualJoint Conference of the IEEE Computer and Communications Societies, 2004.

15. S.Rana and A. Kapil, "Security-Aware Efficient Route Discovery for DSR in MANET," Information and Communication Technologies, Communications in Computer and Information Science, vol. 101, pp. 186-194, 2010.

16. X.Lv and H. Li, "Secure group communication with both confidentiality and non-repudiation for mobile ad-hoc networks," Information Security, IET, vol. 7, 2013. 
17. S.a.A.k.G, H.o.d.R.m, and S. Sharma, "A comprehensive Review of Security Issues in Manets," International Journal of Computer Applications vol. 692013.

18. V.P.and R. P. Goyal, "MANET: Vulnerabilities, Challenges, Attacks, Application," IJCEM International Journal of Computational Engineering \& Management, vol. 11, 2011.

19. A.MISHRA, R. Jaiswal, and S. Sharma, " A novel approach for detecting and eliminating cooperative black hole attack using advanced DRI table in Ad hoc Network," presented at the 3rd International Conference on Advance Computing Conference (IACC), 2013.

20. B.Kannhavong, H. Nakayama, Y. Nemoto, and N. Kato, "A survey of routing attacks in mobile ad hoc networks", Wireless Communications, IEEE Transactions, vol. 14. 